\title{
Argumentation Semantics under a Claim-centric View: Properties, Expressiveness and Relation to SETAFs
}

\author{
Wolfgang Dvořák, Anna Rapberger, Stefan Woltran \\ TU Wien, Institute of Logic and Computation \\ \{dvorak, arapberg, woltran\}@dbai.tuwien.ac.at
}

\begin{abstract}
Claim-augmented argumentation frameworks (CAFs) constitute a generic formalism for conflict resolution of conclusionoriented problems in argumentation. CAFs extend Dung argumentation frameworks (AFs) by assigning a claim to each argument. So far, semantics for CAFs are defined with respect to the underlying AF by interpreting the extensions of the respective $\mathrm{AF}$ semantics in terms of the claims of the accepted arguments; we refer to them as inherited semantics of CAFs. A central concept of many argumentation semantics is maximization, which can be done with respect to arguments as in preferred semantics, or with respect to the range as in semi-stable semantics. However, common instantiations of argumentation frameworks require maximality on the claim-level and inherited semantics often fail to provide maximal claim-sets even if the underlying AF semantics yields maximal argument sets. To address this issue, we investigate a different approach and introduce claim-level semantics (cl-semantics) for CAFs where maximization is performed on the claim-level. We compare these two approaches for five prominent semantics (preferred, naive, stable, semistable, and stage) and relate in total eleven CAF semantics to each other. Moreover, we show that for a certain subclass of CAFs, namely well-formed CAFs, the different versions of preferred and stable semantics coincide, which is not the case for the remaining semantics. We furthermore investigate a recently established translation between well-formed CAFs and SETAFs and show that, in contrast to the inherited naive, semi-stable and stage semantics, the cl-semantics correspond to the respective SETAF semantics. Finally, we investigate the expressiveness of the considered semantics in terms of their signatures.
\end{abstract}

\section{Introduction}

Abstract argumentation frameworks (AFs) as introduced by Dung (1995) provide a general schema for analyzing discourses by treating arguments as abstract entities while an attack relation encodes conflicts between them; the acceptance status of arguments is evaluated with respect to different semantics. Abstract argumentation has been established as an important core formalism for argumentation systems. Depending on the particular task, various instantiation processes are used to model discourses, medical and legal cases (Atkinson et al. 2017), but also logic programs and nonmonotonic reasoning formalisms (Dung 1995; Caminada et al. 2015b).
In a nutshell, an instantiation procedure into AFs includes (1) extraction of arguments and conflicts among them; (2) identification of jointly acceptable arguments (extensions) based on a particular argumentation semantics; (3) inspection of claims of the acceptable arguments in order to draw conclusions about the original system. Different instantiation procedures have been considered, see e.g. ABA (Bondarenko, Toni, and Kowalski 1993), ASPIC (Prakken 2010) or instantiations based on classical logic arguments (Gorogiannis and Hunter 2011). A generalization of AFs which is ideally suited for analyzing instantiation procedures in this spirit - and in a uniform way - are claim-augmented argumentation frameworks (CAFs) which simply extend AFs by assigning a claim to each argument (Dvořák and Woltran 2020).

In this work we reconsider the way $\mathrm{AF}$ semantics are lifted to CAF semantics. A central concept in abstract argumentation semantics are admissible sets, i.e. sets of arguments that defend themselves against all attackers. Preferred semantics for Dung AFs are defined as subset-maximal admissible sets. For CAFs, two natural ways to define preferred semantics come to mind: First, as done in (Dvořák and Woltran 2020), one takes the preferred extensions of the underlying $\mathrm{AF}$ and interprets those in terms of their claims. Second, we interpret all admissible sets of the underlying $\mathrm{AF}$ and select those which are subset-maximal in terms of their claims. We consider the first variant as inherited semantics; the second variant as claim-based semantics, since the claims play a fundamental role in the actual determination of the extensions (while for the inherited variant, standard semantics are just translated into the claims). Similar considerations lead to different variants of other semantics. Hereby, range-based semantics such as stable, semi-stable, and stage semantics require special treatment, since the concept of range (i.e. elements that are attacked by a set of arguments) is now subject of adapting the claim-centric view to the semantics at hand.

Example 1. To illustrate the difference of the two approaches consider the AF given in Figure 1 and assume that $x_{1}$ and $x_{2}$ have assigned claim $x$, the arguments $y_{1}$, $y_{2}$ have claim $y$ and $z$ supports a different claim $z$. The admissible sets are $\emptyset,\left\{y_{1}\right\},\left\{y_{1}, x_{2}\right\},\{z\},\left\{x_{1}, z\right\},\left\{y_{2}, z\right\}$ and $\left\{x_{1}, y_{2}, z\right\}$. Thus the inherited preferred semantics for $C A F$ yields $\{x, y\}$ and $\{x, y, z\}$ while the claim-based pre- 


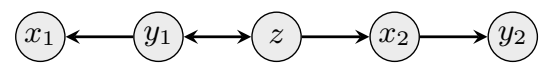

Figure 1: A first example CAF

ferred semantics only results in $\{x, y, z\}$, since only the set $\left\{x_{1}, y_{2}, z\right\}$ is subset-maximal among the admissible sets when interpreted in terms of the arguments' claims.

We thus observe that, in general, inherited and claimbased semantics yield different results. However, as we will see, for an important subclass of CAFs (named well-formed CAFs (Dvořák and Woltran 2020)) that typically arises in many instantiation procedures the two variants of preferred semantics coincide.

Notice that claim-based semantics naturally appear in many instantiations (see e.g. (Caminada et al. 2015a; Caminada et al. 2015b)) where one aims to maximize the accepted/decided claims and not the arguments. The discrepancy between inherited and claim-based preferred semantics is then often circumvented by constructing CAFs under structural restrictions such that inherited and claim-based semantics coincide. However, for range-based semantics the inherited and claim-based versions differ in the standard instantiation procedures and it is even impossible to capture the range-based semantics with an according AF semantics (Caminada et al. 2015a; Caminada et al. 2015b). The additional layer of claims in CAFs provides the right tool to formalize these semantics and study their properties and relations.

In this paper, we introduce claim-based definitions of preferred, naive, stable, semi-stable, and stage semantics and compare these semantics with the corresponding inherited semantics. In particular, we investigate whether these semantics satisfy the fundamental property of I-maximality, i.e., whether the resulting claim-sets are subset-maximal. We consider general CAFs as well as the subclass of wellformed CAF. The latter covers a broad range of fundamental instantiations of argumentation while general CAFs apply to (more advanced) instantiations which allow to take concepts like argument strength or preferences into account. For well-formed CAFs we will show that the inherited and claim-based version of preferred and stable semantics coincide. We then investigate a recently established translation between well-formed CAFs and argumentation frameworks with collective attacks (Dvořák, Rapberger, and Woltran 2020). This translation establishes a one-to-one correspondence for admissible, preferred and stable semantics. Interestingly, as we will show, this result does not extend to the inherited version of naive, semi-stable and stage semantics but to the claim-based version of these semantics. Finally, we compare the expressiveness of all the considered semantics by characterizing their signatures (Dunne et al. 2015) for general and well-formed CAFs. Besides being a measurement for the diversity of view points a semantics can provide in a single framework, signatures are recognized as crucial for operators in dynamics of argumentation (cf. (Baumann and Brewka 2019)).

The main results of our paper are:

- We introduce claim-based definitions for preferred, naive, stable, semi-stable and stage semantics and by that provide argumentation semantics that shift maximization of extensions from argument-level to claim-level.

- We compare claim-based semantics and inherited semantics for CAFs with respect to I-maximality; moreover, we clarify in which way the inherited variant relates to its claim-based counter-part.

- We provide a full picture of the relations between all considered inherited and claim-based semantics for both general and well-formed CAFs.

- We show that the claim-based semantics of well-formed CAFs are in one-to-one correspondence with their counter parts in SETAFs, under the translation of (Dvořák, Rapberger, and Woltran 2020), while inherited semantics are not (unless they coincide with the claim-based version).

- Finally we characterize the signatures of the considered semantics for both general CAFs and well-formed CAFs.

Parts of this paper have been presented at the 9th European Starting AI Researchers' Symposium (STAIRS), see (Rapberger 2020).

\section{Preliminaries}

We introduce argumentation frameworks (Dung 1995); for a comprehensive introduction, see (Baroni, Gabbay, and Giacomin 2018; Baroni, Caminada, and Giacomin 2011). We fix $U$ as countable infinite domain of arguments.

Definition 1. An argumentation framework (AF) is a pair $F=(A, R)$ where $A \subseteq U$ is a finite set of arguments and $R \subseteq A \times A$ is the attack relation. We say that $E \subseteq A$ attacks $b$ if $(a, b) \in R$ for some $a \in E$ and denote by $\bar{E}_{F}^{+}=\{b \in$ $A \mid(a, b) \in R\}$ the set of attacked arguments of $E$. We call $E \cup E_{F}^{+}$the range of $E$ in $F$. An argument $a \in A$ is defended (in $F$ ) by $E \subseteq A$ if $b \in E_{F}^{+}$for each $b$ with $(b, a) \in R$.

Semantics for AFs are defined as functions $\sigma$ which assign to each $\mathrm{AF} F=(A, R)$ a set $\sigma(F) \subseteq 2^{A}$ of extensions. We consider for $\sigma$ the functions $c f$, a dm, naive, stb, prf, sem and $\mathrm{stg}$ which stand for conflict-free, admissible, naive, stable, preferred, semi-stable and stage, respectively.

Definition 2. Let $F=(A, R)$ be an $A F$. A set $E \subseteq A$ is conflict-free (in $F$ ), if there are no $a, b \in E$, such that $(a, b) \in R . \quad c f(F)$ denotes the collection of sets being conflict-free in $F$. For $E \in c f(F)$, we define

- $E \in$ naive $(F)$, if there is no $D \in c f(F)$ with $E \subset D$;

- $E \in \operatorname{adm}(F)$, if each $a \in E$ is defended by $E$ in $F$;

- $E \in \operatorname{prf}(F)$, if $E \in \operatorname{adm}(F)$ and $\nexists D \in \operatorname{adm}(F)$ with $E \subset D$;

- $E \in \operatorname{stb}(F)$, if $E \cup E_{F}^{+}=A$;

- $E \in \operatorname{sem}(F)$, if $E \in \operatorname{adm}(F)$ and $\nexists D \in \operatorname{adm}(F)$ with $E \cup E_{F}^{+} \subset D \cup D_{F}^{+}$;

- $E \in \operatorname{stg}(F)$, if $\nexists D \in c f(F)$, with $E \cup E_{F}^{+} \subset D \cup D_{F}^{+}$.

We recall that for each $\mathrm{AF} F$, stb $(F) \subseteq \operatorname{stg}(F) \subseteq$ naive $(F) \subseteq c f(F)$ and $\operatorname{stb}(F) \subseteq \operatorname{sem}(F) \subseteq \operatorname{prf}(F) \subseteq$ $\operatorname{adm}(F)$; also $\operatorname{stb}(F)=\operatorname{sem}(F)=\operatorname{stg}(F)$ in case $\operatorname{stb}(F) \neq \emptyset$. Moreover, semantics $\sigma \in\{$ naive, prf, stb, 
stg, sem $\}$ deliver incomparable sets, i.e. for all $E, D \in$ $\sigma(F), E \subseteq D$ implies $E=D$; the property is also referred to as I-maximal.

Next we define claim-augmented argumentation frameworks according to Dvořák and Woltran (2020).

Definition 3. A claim-augmented argumentation framework (CAF) is a triple $(A, R$, claim) where $(A, R)$ is an $A F$ and claim : $A \rightarrow C$ is a function which assigns a claim to each argument in $A ; C$ is a set of possible claims. The claimfunction is extended to sets in the following way: For a set $E \subseteq A, \operatorname{claim}(E)=\{\operatorname{claim}(a) \mid a \in E\}$.

A $C A F(A, R$, claim $)$ is called well-formed if $\{a\}_{(A, R)}^{+}=$ $\{b\}_{(A, R)}^{+}$for all $a, b \in A$ such that $\operatorname{claim}(a)=\operatorname{claim}(b)$.

In (Dvořák and Woltran 2020), semantics of CAFs are defined based on the standard semantics of the underlying AF. The extensions are interpreted in terms of the claims of the arguments. We call this variant inherited semantics (isemantics).

Definition 4. For a $C A F C F=(A, R$, claim $)$ and a semantics $\sigma$, we define the i-semantics variant of $\sigma$ as $\sigma_{c}(C F)=$ $\{\operatorname{claim}(E) \mid E \in \sigma((A, R))\}$. We call a set $E \in \sigma((A, R))$ with $\operatorname{claim}(E)=S$ a $\sigma$-realization of $S$ in $C F$.

Basic relations between different semantics carry over from standard AFs, i.e. for any $\mathrm{CAF} C F$, stb $(C F) \subseteq$ $\operatorname{sem}_{c}(C F) \subseteq \operatorname{prf}_{c}(C F) \subseteq a d m_{c}(C F)$ and $s t b_{c}(C F) \subseteq$ $\operatorname{stg}_{c}(C F) \subseteq$ naive $_{c}(C F) \subseteq c f_{c}(C F)$; moreover, if $\operatorname{stb}(C F) \neq \emptyset$ then $\operatorname{stb}_{c}(C F)=\operatorname{sem}_{c}(C F)=\operatorname{stg}_{c}(C F)$. On the other hand observe that we lose fundamental properties of semantics like I-maximality of preferred, naive, stable, semi-stable and stage semantics: Consider the CAF $C F$ from Example 1, then $\operatorname{prf}_{c}(C F)=$ $s^{t} b_{c}(C F)=\operatorname{sem}_{c}(C F)=\operatorname{stg}_{c}(C F)=\{\{x, y\},\{x, y, z\}\}$ and naive $_{c}(C F)=\{\{x\},\{y\},\{x, y\},\{x, y, z\}\}$. Note that $C F$ is not well-formed.

In the remainder of the section, we provide a few definitions in order to deal with the concept of range on the claim level which we will use to define our new versions for stable, semi-stable, and stage semantics.

Definition 5. Let $C F=(A, R$, claim $), E \subseteq A$ and $c \in$ $\operatorname{claim}(A)$. We say that $E$ defeats $c$ (in $C F$ ) iff $E$ attacks (in $(A, R)$ ) every $a \in A$ with $\operatorname{claim}(a)=c$. We define $\nu_{C F}(E)=\{c \in \operatorname{claim}(A) \mid E$ defeats $c$ in $C F\}$.

Observe that $\nu_{C F}: A \rightarrow \operatorname{claim}(A)$ is monotone, i.e. if $E \subseteq D$ then $\nu_{C F}(E) \subseteq \nu_{C F}(D)$ for any $E, D \subseteq A$. Moreover, for each well-formed CAF $C F=(A, R$, claim $)$, the set of defeated claims $\nu_{C F}(E)$ is determined by the claims which appear in $E$ since $E_{(A, R)}^{+}=D_{(A, R)}^{+}$for all $E, D \subseteq A$ with $\operatorname{claim}(E)=\operatorname{claim}(D)$.

Lemma 1. Let $C F=(A, R$, claim $)$ be well-formed and let $E, D \subseteq A$ with $\operatorname{claim}(E)=\operatorname{claim}(D)$, then $\nu_{C F}(E)=$ $\nu_{C F}(\bar{D})$.

Thus the concept of range is easily adaptable to claim-sets in well-formed CAFs.

Definition 6. For a well-formed $C A F C F$, for $S \subseteq$ $\operatorname{claim}(A)$, we define $S_{C F}^{+}=\nu_{C F}(E)$ for some $E \subseteq A$ with $\operatorname{claim}(E)=S$. We call $S \cup S_{C F}^{+}$the range of $S$ in $C F$.
However, in general CAFs, different realizations of a claim-set $S$ might yield different sets of defeated claims. Thus, for a semantics $\sigma$, we define the set $\mathcal{N}_{\sigma}^{C F}(S)$ which contains $\nu_{C F}(E)$ for each $\sigma$-realization $E$ of $S$.

Definition 7. For a CAF $C F=(A, R$, claim $), S \subseteq$ $\operatorname{claim}(A)$ and a semantics $\sigma$, let $\mathcal{N}_{\sigma}^{C F}(S)=\left\{\nu_{C F}(E) \mid\right.$ $E \in \sigma((A, R)), \operatorname{claim}(E)=S\}$. For each $S^{\prime} \in \mathcal{N}_{\sigma}^{C F}$, we call $S \cup S^{\prime}$ a range of $S$ in $C F$.

\section{Comparing Semantics}

In this section we provide new variants for preferred, naive, stable, semi-stable, and stage semantics; in fact, we will have two new versions of stable semantics. In each of the subsequent subsections, the new claim-based semantics is compared to its inherited counterpart and we investigate whether I-maximality holds. Both properties are analyzed for general and well-formed CAFs.

\subsection{Preferred Semantics}

We introduce preferred semantics for CAFs which yield $\subseteq$ maximal admissible claim-sets, that is, we consider maximization on claim-level (cl-preferred semantics).

Definition 8. Let $C F=(A, R, \operatorname{claim})$ and $S \subseteq \operatorname{claim}(A)$. Then $S$ is a cl-preferred claim-set $(S \in \operatorname{cl}$-prf $(C F))$ iff $S \in \operatorname{adm} m_{c}(C F)$ and there is no $T \in \operatorname{adm}_{c}(C F)$ with $S \subset T$.

We show that cl-preferred semantics constitutes a strengthening of i-preferred semantics, that is, we show that each cl-preferred claim-set is also i-preferred.

Proposition 1. $c l-p r f(C F) \subseteq \operatorname{prf} f_{c}(C F)$ for each $C A F C F$.

Proof. Let $C F=(A, R$, claim). Given $S \in \operatorname{cl}$-prf $(C F)$, we show that $S$ has a maximal $a d m$-realization $E$ in $(A, R)$. Else there is a (maximal) $D \in \operatorname{adm}((A, R))$ such that $E \subset$ $D$ and $\operatorname{claim}(D) \neq \operatorname{claim}(E)$. But then $S \subset \operatorname{claim}(D)$ by monotonicity of the claim-function; contradiction.

The other direction does not hold: In Example 1, $\operatorname{prf}_{c}(C F)=\{\{x, y\},\{x, y, z\}\}$ but $\operatorname{cl}-\operatorname{prf}(C F)=$ $\{\{x, y, z\}\}$. In fact, there is no CAF which realizes $\{\{x, y\},\{x, y, z\}\}$ under cl-preferred semantics, since, by definition, this semantics yields I-maximal claim-sets.

Proposition 2. For every $C A F C F=(A, R$, claim $)$, cl-prf $(C F)$ is I-maximal.

We show next that for well-formed CAFs, $\operatorname{prf}_{c}$ and $c l$-prf semantics coincide. The following lemma is crucial.

Lemma 2. Let $C F=(A, R$, claim $)$ be a well-formed $C A F$, $E, D \in \operatorname{prf}((A, R)), E \neq D$. Then $\operatorname{claim}(E) \nsubseteq \operatorname{claim}(D)$.

Proof. First assume, there exists an $a \in E$ attacking some $b \in D$ in $(A, R)$. It follows that $\operatorname{claim}(a) \notin \operatorname{claim}(D)$, otherwise the argument $c \in D$ with $\operatorname{claim}(c)=\operatorname{claim}(a)$ also attacks $b$ due to well-formedness; since $D$ is conflictfree, this cannot be the case. Suppose now that no $a \in E$ attacks some $b \in D$. We need at least one attack $(a, b)$ from $E$ to $D$, otherwise $E \cup D \in \operatorname{prf}((A, R))$. But then $E$ needs to attack $b$ since $E$ is admissible, so we are done. 


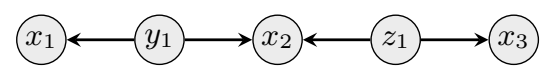

Figure 2: The AF from Example 2.

Proposition 3. $c l-p r f(C F)=\operatorname{prf}_{c}(C F)$ for each wellformed $C A F C F$.

Proof. We show that $\operatorname{prf}_{c}(C F) \subseteq$ cl-prf $(C F)$ (cf. Proposition 1 for the other direction): Consider a set $S \in \operatorname{prf}_{c}(C F)$ and its $p r f$-realization $E$ in $C F$. Then $S$ is maximal among $a d m_{c}(C F)$ wrt. subset-relation: Towards a contradiction, assume that there is a claim-set $T \in a d m_{c}(C F)$ such that $T \supset S$. Consider its $a d m$-realization $E^{\prime} \in \operatorname{adm}((A, R))$. But then there is also a preferred extension $E^{\prime \prime} \supseteq E^{\prime}$ with $S \subset T \subseteq \operatorname{claim}\left(E^{\prime \prime}\right)$, contradiction to Lemma 2 .

It follows that for well-formed CAFs, also i-preferred semantics yield I-maximal claim-sets. Moreover, by Lemma 2, each i-preferred claim-set has a unique prfrealization in the underlying AF.

Proposition 4. For every well-formed $C A F C F=$ $(A, R$, claim $)$, we have (1) $\operatorname{prf}_{c}(C F)$ satisfies I-maximality, and (2) $|\operatorname{prf}((A, R))|=\left|\operatorname{prf}_{c}(C F)\right|$.

\subsection{Naive Semantics}

We introduce cl-naive semantics for CAFs which shift maximization of conflict-free sets from argument-level to claimlevel. We show that, similar to the relation between clpreferred and i-preferred semantics, each cl-naive claim-set is also i-naive; although, in contrast to preferred CAF semantics, even for well-formed CAFs, both versions of naive CAF semantics potentially yield different claim-sets.

Definition 9. Let $C F=(A, R, \operatorname{claim})$ and $S \subseteq \operatorname{claim}(A)$. Then $S$ is a cl-naive claim-set $(S \in$ cl-naive $(C F))$ iff $S \in$ $c f_{c}(C F)$ and there is no $T \in c f_{c}(C F)$ with $S \subset T$.

We show that each cl-naive claim-set is i-naive.

Proposition 5. cl-naive $(C F) \subseteq$ naive $_{c}(C F)$ for each $C A F$ $C F$.

Proof. Let $S \in$ cl-naive $(C F)$. We show that $S$ has a maximal $c f$-realization $E$ in $(A, R)$. Else there is a (maximal) conflict-free set $D \subseteq A$ such that $E \subset D$ and $\operatorname{claim}(D) \neq$ $\operatorname{claim}(E)$. But then $S \subset \operatorname{claim}(D)$ by monotony of the claim-function, contradiction to the maximality of $S$.

Similarly to cl-preferred semantics, we have that the other direction does not hold in general since, in contrast to i-naive semantics, cl-naive semantics yield I-maximal claim-sets.

Proposition 6. For every $C A F C F=(A, R$, claim $)$, cl-naive $(C F)$ is I-maximal.

The next example shows that even for well-formed CAFs, I-maximality for i-naive semantics is not guaranteed.

Example 2. Let $C F=(A, R$, claim $)$ with $(A, R)$ as in Figure 2, $\operatorname{claim}\left(x_{i}\right)=x$ for $i \leq 3, \operatorname{claim}\left(y_{1}\right)=y$ and $\operatorname{claim}\left(z_{1}\right)=z$. Note that $C F$ is indeed well-formed. Then naive $_{c}(C F)=\{\{x\},\{x, y\},\{x, z\},\{y, z\}\}$.

By the above example we obtain that naive $e_{c}$ and cl-naive semantics differ even on well-formed CAFs.

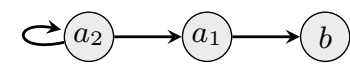

Figure 3: Example of a $\mathrm{CAF} C F=(A, R$, claim $)$ with $\operatorname{claim}\left(a_{1}\right)=\operatorname{claim}\left(a_{2}\right)=a, \operatorname{claim}(b)=b$.

\subsection{Stable Semantics}

We introduce two variants of stable semantics based on maximization on claim-level. The first variant requires the underlying realization of a claim-set $S$ to be conflict-free, while the second variant requires admissibility. We clarify the relation between both variants as well as the relation to i-stable semantics and compare them also with regard to Imaximality of their claim-sets.

Definition 10. Let $C F=(A, R$, claim $)$ and $S \subseteq$ $\operatorname{claim}(A) . S$ is a cl-stable claim-set $\left(S \in\right.$ cl-stb $\left._{c f}(C F)\right)$ iff there exists $S^{\prime} \in \mathcal{N}_{c f}^{C F}(S)$ such that $S \cup S^{\prime}=\operatorname{claim}(A)$.

The proposed variant of claim-based stable semantics relaxes the definition of inherited stable semantics in the way that it is no longer required that a $s t b$ realization of a cl-stable claim-set exists. Consider the CAF $C F=(A, R, \operatorname{claim})$ from Figure 3 with $\operatorname{claim}\left(a_{1}\right)=$ $\operatorname{claim}\left(a_{2}\right)=a, \operatorname{claim}(b)=b$. Here, $\operatorname{stb}_{c}(C F)=\emptyset$ but $c l$-stb $b_{c f}(C F)=\{\{a\}\}$ : The $c f$-realization $E=\left\{a_{1}\right\}$ satisfies $\nu_{C F}(E)=\{b\}$ and therefore, $\operatorname{claim}(E) \cup \nu_{C F}(E)=$ $\operatorname{claim}(A)$. Observe that $C F$ is not well-formed. Furthermore notice that the cl-stable claim-set $\{a\}$ is in fact not $a d m$-realizable in $(A, R)$. Thus in contrast to standard $\mathrm{AF}$ semantics where each stable extension satisfies admissibility, we have that a $c l$-stb-realization in the underlying $\mathrm{AF}$ is not necessarily admissible. We consider therefore also a stronger notion of stable semantics which requires $a d m$ realizability in the underlying $\mathrm{AF}$.

Definition 11. Let $C F=(A, R$, claim $)$ and $S \subseteq$ $\operatorname{claim}(A) . S$ is an adm-cl-stable set $(S \in \mathrm{cl}$-stb adm $(C F))$ if there exists $S^{\prime} \in \mathcal{N}_{a d m}^{C F}(S)$ such that $S \cup S^{\prime}=\operatorname{claim}(A)$.

Proposition 7. For any $C F=(A, R$, claim $), ~ s t b_{c}(C F) \subseteq$ $c l-s t b_{a d m}(C F) \subseteq c l-s t b_{c f}(C F)$.

Proof. We first show $s t b_{c}(C F) \subseteq c l-s t b_{a d m}(C F)$ : Let $S \in$ $s t b_{c}(C F)$ and consider a $s t b$-realization $E \subseteq A$ (observe that $E \in \operatorname{adm}((A, R)))$. Let $c \in \operatorname{claim}(A) \backslash S$, then for all $x \in A$ with $\operatorname{claim}(x)=c, x \in A \backslash E$. Since $E$ is stable in $(A, R)$ we have that $E$ attacks each argument $x \in A \backslash E$, therefore $c \in \nu_{C F}(E)$. Thus $\nu_{C F}(E)=\operatorname{claim}(A) \backslash S$, i.e. we have found a set $S^{\prime}=\nu_{C F}(E) \in \mathcal{N}_{a d m}^{C F}(S)$ with $S \cup S^{\prime}=\operatorname{claim}(A)$, that is, $S \in c l$-stb adm $(C F)$. To show $c l$-stb $b_{a d m}(C F) \subseteq c l$-stb ${ }_{c f}(C F)$, observe that for each claim-set $S, \mathcal{N}_{a d m}^{C F}(S) \subseteq \mathcal{N}_{c f}^{C F}(S)$ : Indeed, if $\nu_{C F}(E) \in$ $\mathcal{N}_{a d m}^{C F}(S)$ for some $E \subseteq A$, then $E \in \operatorname{adm}((A, R)) \subseteq$ $c f((A, R))$, and thus $\nu_{C F}(E) \in \mathcal{N}_{c f}^{C F}(S)$.

The CAF $C F=(A, R$, claim $)$ from Figure 3 shows that $c l$-stb ${ }_{a d m}(C F) \neq c l$-stb $c f(C F)$ since $c l$-stb $a d m(C F)=\emptyset$ but $c l-s t b_{c f}(C F)=\{\{a\}\}$. A small modification of the CAF $C F$ shows that $c l$-stb adm $(C F) \neq s t b_{c}(C F)$ : Let $C F_{1}=\left(A, R \backslash\left\{\left(a_{2}, a_{1}\right)\right\}\right.$, claim $)$, then $c l$-stb $a d m\left(C F_{1}\right)=$ 
$\{\{a\}\}$ (witnessed by the adm-realization $\left\{a_{1}\right\}$ in $(A, R)$ ) but $s t b_{c}\left(C F_{1}\right)=\emptyset$. Observe that both $C F$ and $C F_{1}$ are not well-formed. We will show next that for well-formed CAFs, all considered variants of stable semantics are in fact equal.

Proposition 8. For any well-formed $C A F C F=(A, R$,

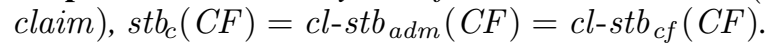

Proof. We will show that $c l-s t b_{c f}(C F) \subseteq s t b_{c}(C F)$, the result then follows immediately from Proposition 7.

Let $S \in c l$-stb $b_{c f}(C F)$, then $S \cup S_{C F}^{+}=\operatorname{claim}(A)$ (recall that by Lemma $1, S_{C F}^{+}=\nu_{C F}(E)=\nu_{C F}(D)$ for any $E, D \subseteq A$ with $\operatorname{claim}(E)=\operatorname{claim}(D)=S$ ). We consider a maximal $c f$-realization $E \subseteq A$ of $S$, that is, $E \in c f((A, R))$ with $E=\operatorname{claim}(S)$ and for every set $D \in c f((A, R))$ with $D=\operatorname{claim}(S), D \subseteq E$. We show that $E_{(A, R)}^{+}=A \backslash E$. Let $x \in A \backslash E$ and let $\operatorname{claim}(x)=c$. If $c \notin S$, then $c \in S_{C F}^{+}$by definition of cl-stable semantics, thus $E$ attacks $x$. Consider now the case $c \in S$, i.e. there is an argument $y \in E$ such that $\operatorname{claim}(y)=c$ and observe that $E \cup\{x\}$ is not conflict-free by maximality of $E$; thus either (a) $(x, x) \in R$ or there is $z \in E$ such that either (b) $(z, x) \in R$ or (c) $(x, z) \in R$. In case (a) then also $(y, x) \in R$ by well-formedness; in case (b) we are done; in case (c) we have $(y, z) \in R$ by well-formedness and therefore $E$ is not conflict-free, contradiction.

Recall that i-stable claim-sets are not I-maximal in general (cf. Example 1). As a consequence of Proposition 7 we deduce that also cl-stable claim-sets are not I-maximal. For well-formed $C F$ we have that $s t b_{c}(C F)$ is I-maximal, as $\operatorname{prf}_{c}(C F)$ is I-maximal (Proposition 4) and $s t b_{c}(C F) \subseteq$ $\operatorname{prf}_{c}(C F)$. By Proposition 8, we have that cl-stable claimsets satisfy I-maximality if well-formedness is guaranteed.

Proposition 9. For each well-formed $C A F C F$, stb $b_{c}(C F)$, $c l$-stb $b_{c f}(C F)$ and $c l-s t b_{a d m}(C F)$ are I-maximal.

\subsection{Semi-stable Semantics}

We consider the following claim-based variant of semistable semantics which relaxes $c l-s t b_{a d m}$ semantics by dropping the requirement that the range of a claim-set must consist of all claims in the framework. Instead, we consider claim-sets with maximal range.

Definition 12. Let $C F=(A, R, \operatorname{claim}), S \subseteq \operatorname{claim}(A)$ is a cl-semi-stable claim-set, in symbols $S \in \mathrm{cl}$-sem $(C F)$, iff there exists $S^{\prime} \in \mathcal{N}_{\text {adm }}^{C F}(S)$ such that there is no $T \in$ $a d m_{c}(C F), T^{\prime} \in \mathcal{N}_{a d m}^{C F}(T)$ with $S \cup S^{\prime} \subset T \cup T^{\prime}$.

Notice that for well-formed CAFs, the definition reduces to checking $\subseteq$-maximality of $S \cup S_{C F}^{+}$for i-admissible claim-sets $S$ since the range of $S$ is unique in this case.

In contrast to the semantics we considered so far, we observe that the proposed variant of semi-stable semantics neither constitutes a strengthening nor a weakening of its inherited counterpart. The following example shows that even for well-formed CAFs, cl-semi-stable and i-semi-stable semantics potentially yield different claim-sets.

Example 3. Consider the well-formed $C A F C F$ from Figure 4 with $\operatorname{claim}\left(b_{i}\right)=b$, $\operatorname{claim}\left(f_{i}\right)=f$ and $\operatorname{claim}(x)=x$

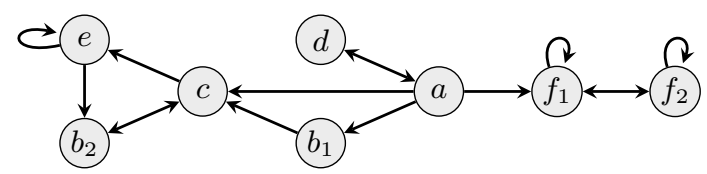

Figure 4: CAF $C F$ from Example 3.

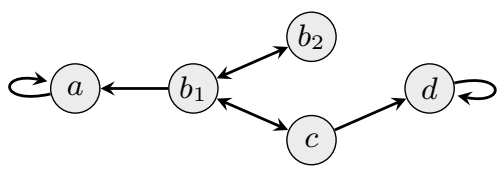

Figure 5: CAF CF from Example 4.

for $x \in\{a, c, d, e\}$. In order to evaluate $C F$ with respect to cl-semi-stable semantics, first consider non-empty $i$-admissible claim-sets which are given by $S_{1}=\{d\}, S_{2}=$ $\{b, d\}$ and $S_{3}=\{a\}$; moreover, $S_{1, C F}^{+}=\{a\}, S_{2, C F}^{+}=$ $\{a, c\}$ and $S_{3, C F}^{+}=\{c, d\}$; thus cl-sem $(C F)=\{\{b, d\}\}$. Observe that $\{a\}$ is the only $i$-semi-stable claim-set.

Example 3 shows that cl-semi-stable and i-semi-stable semantics are incomparable; nevertheless, they admit similar behavior when it comes to I-maximality of their claimsets. Recall that i-semi-stable claim-sets are in general not I-maximal; the following example shows that this is also the case for cl-semi-stable semantics.

Example 4. Consider the $C A F C F=(A, R$, claim $)$ from Figure 5 with $\operatorname{claim}\left(b_{1}\right)=\operatorname{claim}\left(b_{2}\right)=b$ and $\operatorname{claim}(x)=$ $x$ for $x \in A \backslash\left\{b_{1}, b_{2}\right\}$. First notice that $\operatorname{stb}_{c}(C F)=$ $c l$-stb $b_{c f}(C F)=c l$-stb $b_{a d m}(C F)=\emptyset$ since $b_{1}$ and care mutually attacking, thus either a or d are not attacked. The nonempty inherited admissible sets are $S_{1}=\{b\}, S_{2}=\{c\}$ and $S_{3}=\{b, c\}$; then $\mathcal{N}_{a d m}\left(S_{1}\right)=\{\{\emptyset,\{a, c\}\}$ and $\mathcal{N}_{\text {adm }}\left(S_{2}\right)=\mathcal{N}_{\text {adm }}\left(S_{3}\right)=\{\{d\}\}$. Observe that $S_{2}$ is not cl-semi-stable, since $S_{2} \cup\{d\} \subseteq S_{3} \cup\{d\}$; moreover, $S_{1}$ is cl-semi-stable, since $S_{1} \cup\{a, c\}=\{a, b, c\} \nsubseteq S_{2} \cup\{d\}, S_{3}$ is cl-semi-stable, since $S_{3} \cup\{d\}=\{b, c, d\} \nsubseteq S_{1}$.

Notice that the CAF $C F$ in Example 4 is not well-formed. In fact, on well-formed CAFs both cl-semi-stable and isemi-stable semantics yield I-maximal claim-sets.

Proposition 10. For each well-formed $C A F C F$, cl-sem $(C F)$ and $\operatorname{sem}_{c}(C F)$ are I-maximal.

Proof. I-maximality of $c l$-sem $(C F)$ follows by Lemma 1.

To show that $\operatorname{sem}_{c}(C F)$ is I-maximal for each wellformed $C F=(A, R$, claim $)$, let $F=(A, R)$ and assume that there are two semi-stable claim-sets $S, T \in \operatorname{sem}_{C}(C F)$ such that $S \subset T$. We consider sem-realizations $E, D$ for $S, T$ respectively. First, observe that $E_{F}^{+} \subseteq D_{F}^{+}$holds by well-formedness: Let $x \in E_{F}^{+}$, then there is $y \in E$ such that $(y, x) \in R$. By assumption $S \subseteq T$, there exists $z \in D$ such that $\operatorname{claim}(y)=\operatorname{claim}(z)$, thus $(z, x) \in R$ by well-formedness. Second, since semi-stable extensions are I-maximal on the argument level, there is at least one $u \in E \backslash D$. By $E_{F}^{+} \subseteq D_{F}^{+}, u$ is defended by $D$ in $F$. Thus, $D \cup\{u\} \in \operatorname{adm}(F)$ and $D \cup\{u\} \cup(D \cup\{u\})_{F}^{+} \supset D \cup D_{F}^{+}$; contradiction to $D$ being semi-stable. 


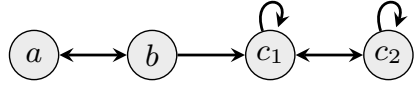

Figure 6: CAF $C F$ from Example 5.

\subsection{Stage Semantics}

We next define cl-stage semantics in the same spirit as clsemi-stable semantics.

Definition 13. Let $C F=(A, R$, claim), then $S \subseteq$ $\operatorname{claim}(A)$ is a cl-stage claim-set, in symbols $S \bar{\epsilon}$ cl-stg $(C F)$, there exists $S^{\prime} \in \mathcal{N}_{c f}^{C F}(S)$ such that there is no $T \in c f_{c}(C F), T^{\prime} \in \mathcal{N}_{c f}^{C F}(T)$ with $S \cup S^{\prime} \subset T \cup T^{\prime}$.

Similarly to cl-semi-stable semantics, cl-stage and i-stage semantics are incomparable. We provide examples of CAFs where cl-stage and i-stage semantics yield a different output. Observe that the employed CAFs are indeed well-formed.

Example 5. Let $C F=(A, R$, claim $)$ with $(A, R)$ given in Figure 6, $\operatorname{claim}\left(c_{1}\right)=\operatorname{claim}\left(c_{2}\right)=c, \operatorname{claim}(a)=a$ and claim $(b)=b$. Then $\{b\}$ is the only $i$-stage claim-set. Consider now the cl-stage claim-sets: The conflict-free sets are $\{a\}$ and $\{b\}$; inspecting the range yields cl-stg $(C F)=$ $\{\{a\},\{b\}\}$ and thus cl-stg $(C F) \not \operatorname{stg}_{c}(C F)$.

Example 6. We modify the CAF CF from Example 5: Let $C F^{\prime}=\left(A^{\prime}, R^{\prime}\right.$, claim $)$ with $A^{\prime}=A \cup\left\{d_{1}, d_{2}\right\}, R^{\prime}=R \cup$ $\left\{\left(d_{1}, d_{2}\right),\left(d_{2}, d_{2}\right),\left(b, d_{1}\right)\right\}$ and claim $\left(d_{i}\right)=d$ for $i \leq 2$. Then $\operatorname{stg}_{c}(C F)=\{\{a, d\},\{b\}\}$ but $\{a, d\}$ is the only clstage claim-set, i.e. $\operatorname{stg}_{c}\left(C F^{\prime}\right) \nsubseteq \mathrm{cl}$-stg $\left(C F^{\prime}\right)$.

Recall that i-stage semantics do not satisfy I-maximality in general (cf. Example 1). The CAF $C F$ from Figure 5 (note that $c l$-sem $(C F)=c l-s t g(C F)$ ) shows that also for cf-stage semantics, I-maximality does not hold for arbitrary CAFs . However, for well-formed CAFs, I-maximality is guaranteed for both cl-stage and i-stage semantics.

Proposition 11. For each well-formed $C A F C F$, both cl-stg $(C F)$ and $\operatorname{stg}_{c}(C F)$ are I-maximal.

Proof. I-maximality of $c l-s t g(C F)$ follows from Lemma 1. To show that $\operatorname{stg}_{c}(C F)$ is I-maximal for each well-formed $C F=(A, R$, claim $)$, let $F=(A, R)$ and assume that there are $S, T \in \operatorname{stg}_{c}(C F)$ such that $S \subset T$. Consider $s t g$ realizations $E, D$ of $S$ and $T$, respectively, that is, $E \cup E_{F}^{+}$, $D \cup D_{F}^{+}$are incomparable and both subset-maximal. Observe that $E_{F}^{+} \subseteq D_{F}^{+}$by well-formedness. Therefore we have that $E_{F}^{+} \subseteq D \cup D_{F}^{+}$, consequently, it must be the case that $E \not \subset D \cup D_{F}^{+}$, i.e. there exists $a \in E$ such that $a \notin D$ and $a \notin D_{F}^{+}$. Let $D^{\prime}=D \cup\{a\}$, then (i) $D^{\prime}$ is conflictfree since $a \notin D_{F}^{+}$and $a$ does not attack $D$ (assume otherwise, then there is some $b \in D$ such that $b \in E_{F}^{+}$, but then also $b \in D_{F}^{+}$since $E_{F}^{+} \subseteq D_{F}^{+}$, contradiction) and, furthermore, $(a, a) \notin R$ since $a \in E$; (ii) $D_{F}^{\prime+}=D_{F}^{+}$since $\operatorname{claim}(a) \in \operatorname{claim}(D)$. Thus we have found a conflict-free set $D^{\prime} \subseteq A$ such that $D^{\prime} \cup D_{F}^{\prime+} \supset D \cup D_{F}^{+}$, contradiction to the subset-maximality of $D \cup D_{F}^{+}$.

\begin{tabular}{l|cccc} 
& \multicolumn{2}{|c}{ CAFs } & \multicolumn{2}{c}{ well-formed CAFs } \\
& Relation & I-max & Relation & I-max \\
\hline prf $_{c} /$ cl-prf & $\supseteq$ & x / & $=$ & $\checkmark / \checkmark$ \\
naive $_{c} /$ cl-naive & $\supseteq$ & x / & $\supseteq$ & x / $\checkmark$ \\
stb $_{c} /$ cl-stb & $\subseteq$ & $\mathrm{x} / \mathrm{x}$ & $=$ & $\checkmark / \checkmark$ \\
sem $_{c} /$ cl-sem & - & $\mathrm{x} / \mathrm{x}$ & - & $\checkmark / \checkmark$ \\
stg $_{c} /$ cl-stg & - & $\mathrm{x} / \mathrm{x}$ & - & $\checkmark / \checkmark$
\end{tabular}

Table 1: Comparison of different approaches to define semantics.

\subsection{Summary}

The results of this section are summarized in Table 1. For each pair of the five semantics $\sigma$ considered $(\tau \in\{c f, a d m\}$ for the two cl-stable variants), the corresponding row provides the results (i) in which way the inherited semantics $\sigma_{c}$ relates to the claim-based semantics $c l-\sigma$ (the relation symbol $R$ in the cell indicates whether for each (wellformed) $C F, \sigma_{c}(C F) R c l-\sigma(C F)$ holds; "-" indicates that $\sigma_{c}(C F)$ and $c l-\sigma(C F)$ are incomparable) and (ii) whether I-maximality holds.

\section{Relations between Semantics}

We first state a general observation which clarifies the relation between inherited and claim-level semantics in case every argument possesses a unique claim. In that case, both variants coincide with the standard AF semantics.

Lemma 3. For any $\sigma \in\{$ prf, naive, stb, sem, stg $\}$ and $C A F C F=(A, R$, claim $)$ with claim $(a)=$ a for all $a \in A$, we have cl- $\sigma(C F)=\sigma_{c}(C F)=\sigma((A, R))$.

It follows that negative results (via counter-examples) showing that two AF semantics $\sigma, \tau$ are not in a subsetrelation immediate apply to (well-formed) CAFs.

Theorem 1. The relations between the semantics depicted in Figure 7 for general CAFs and in Figure 8 for well-formed CAFs hold.

As already discussed in Section 2 the relations between inherited semantics follow from the corresponding relations for Dung AFs. Moreover, in Section 3 the relations between semantics that are based on the same Dung semantics have been settled. We next show the remaining $\subseteq$-relations. First, for any CAF $C F$ and $S \in c l-s t b_{a d m}(C F)$ by definition there is $S^{\prime} \in \mathcal{N}_{a d m}^{C F}(S)$ such that $S \cup S^{\prime}=A$ and thus $S \in c l$-sem $(C F)$, i.e. $c l$-stb $a d m(C F) \subseteq c l$-sem $(C F)$. A similar reasoning applies for the $c f$-based counter-parts, i.e. for every $S \in c l-s t b_{c f}(C F)$ there is a $S^{\prime} \in \mathcal{N}_{c f}^{C F}(S)$ such that $S \cup S^{\prime}=A$ and thus $S \in \operatorname{cl}$-stg $(C F)$, i.e. $c l-s t b_{c f}(C F) \subseteq c l-s t g(C F)$. The positive results for general CAFs are completed by the following lemma.

Lemma 4. For each CAF CF, it holds that (i) cl-sem $(C F) \subseteq \operatorname{prf}_{c}(C F)$, (ii) cl-stg $(C F) \subseteq \operatorname{naive}_{c}(C F)$.

Proof. (i) Let $C F=(A, R$, claim $), S \in \operatorname{cl-sem}(C F)$ and let $E \subseteq A$ such that $\operatorname{claim}(E)=S$ and $\nu_{C F}(E)=S^{\prime}$ such that $S \cup S^{\prime}$ is maximal. Towards a contradiction, assume that $E \notin \operatorname{prf}_{c}(C F)$. Then there exists non-empty $D \subseteq A$ such that $E \cup D \in \operatorname{adm}((A, R))$ and $\operatorname{claim}(D) \nsubseteq S$. As $E \cup D$ is conflict-free, we have $\operatorname{claim}(D) \cap \nu_{C F}(E)=\emptyset$, and thus, 


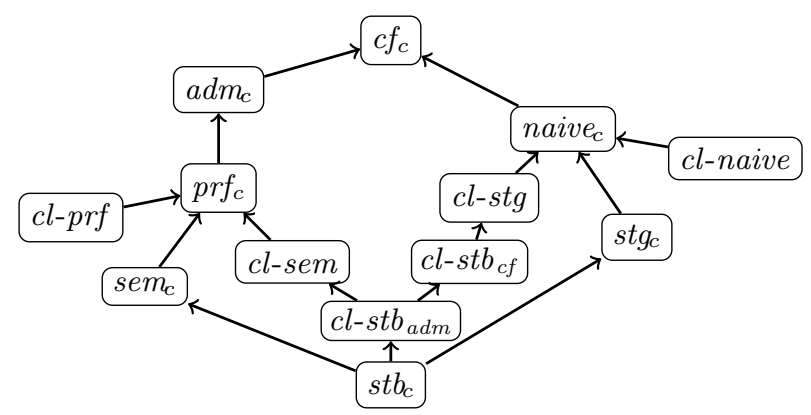

Figure 7: Relations between semantics for CAFs. An arrow from $\sigma$ to $\tau$ indicates that $\sigma(C F) \subseteq \tau(C F)$ for each CAF $C F$.

by monotonicity of $\nu_{C F}$, that $S \cup S^{\prime} \subset \operatorname{claim}(E \cup D) \cup$ $\nu_{C F}(E \cup D)$; contradiction to $S \in \mathrm{cl}$-sem $(C F)$.

(ii) is by a similar argument.

The positive results for well-formed CAFs are completed by the following lemma.

Lemma 5. For each well-formed $C A F C F$, the following relations hold: (i) cl-stg $(C F) \subseteq$ cl-naive $(C F)$; (ii) $\operatorname{stg}_{c}(C F) \subseteq$ cl-naive $(C F)$.

Proof. (i) Assume $S \in$ cl-stg $(C F)$ and $S \notin$ cl-naive $(C F)$, i.e. there is $T \in c f_{c}(C F)$ with $T \supset S$. Then also $T \cup T_{C F}^{+} \supset$ $S \cup S_{C F}^{+}$; contradiction to the maximality of $S \cup S_{C F}^{+}$.

(ii) Let $C F=(A, R$, claim $)$ be well-formed and let $S \in$ $\operatorname{stg}_{c}(C F)$, i.e. there is a set $E \subseteq A$ with $\operatorname{claim}(E)=S$ such that $E \cup E_{F}^{+}$is maximal wrt. subset-relation. Now, assume that $S \notin c l$-naive $(C F)$, i.e. there exists a set $T \in c f_{c}(C F)$ such that $T \supset S$. For each $c f$-realization $D$ of $T$, there is $x \in E \cup E_{F}^{+}$such that $x \notin D \cup D_{F}^{+}$(by maximality of $E \cup E_{F}^{+}$). Since $C F$ is well-formed and $T \subset S$ we have that $D_{F}^{+} \supseteq E_{F}^{+}$. Consequently, we have $x \in E$ and $x \notin D$ We can assume that $x$ and $D$ are conflicting; otherwise consider $D^{\prime}=D \cup\{x\}$ instead. Since $x$ and $D$ are conflicting and since $x \notin D_{F}^{+}$, there exists $y \in D$ such that $(x, y) \in R$. Since $T \subset S$, there is $z \in D$ such that $\operatorname{claim}(x)=\operatorname{claim}(z)$. By well-formedness, $(z, y) \in R$, contradiction to $D$ being conflict-free.

We discuss counter-examples for the remaining cases: The absence of a relation between $c l-\operatorname{sem}(C F)$ and $\operatorname{sem}_{c}(C F)$, where $C F$ is well-formed, is by Example 3; similar, for $c l-s t g(C F)$ and $s t g_{c}(C F)$ by Example 5 and Example 6. Counter-examples for the relations of stable semantics in general CAFs have been discussed after Proposition 7. The absence of relations between $\mathrm{sem}_{c}, \mathrm{cl}$-sem and $\mathrm{cl}$-prf $\left(s t g_{c}, c l\right.$-stg and cl-naive respectively) is by the fact that cl-prf (cl-naive) satisfies I-maximality while the other semantics do not (cf. Figure 5). Finally, all the other cases have counter-examples for Dung AFs and thus, by Lemma 3, also for CAFs.

Recall that for inherited semantics, $s t b_{c}(C F)=$ $\operatorname{sem}_{c}(C F)=\operatorname{stg}_{c}(C F)$ in case $s t b_{c}(C F) \neq \emptyset$. One can show that this does not extend to cl-stable semantics. However, we can obtain the following weaker version.

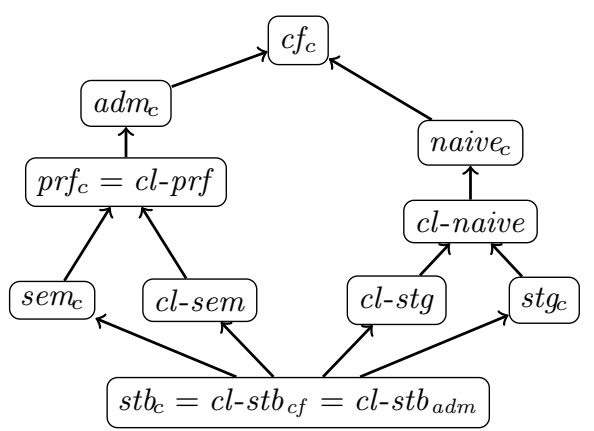

Figure 8: Relations between semantics for well-formed CAFs. An arrow from $\sigma$ to $\tau$ indicates that $\sigma(C F) \subseteq \tau(C F)$ for each wellformed CAF $C F$.

Lemma 6. For any $C A F C F$, (a) $c l$-st $b_{c f}(C F) \neq \emptyset$ implies $c l$-stb $b_{c f}(C F)=c l$-stg $(C F)$ and $(b) c l$-stb adm $(C F) \neq \emptyset$ implies cl-stb $b_{a d m}(C F)=c l$-sem $(C F)$.

\section{Relating well-formed CAFs and SETAFs}

AFs with collective attacks (SETAFs), as introduced by Nielsen and Parsons (2006), generalize the binary attack-relation in AFs to collective attacks of arguments. In (Dvořák, Rapberger, and Woltran 2020) a strong relation between well-formed CAFs and SETAFs has been established. That is, there is a translation from well-formed CAFs to SETAFs (and vice versa) such that $c f_{c}, a d m_{c}, s t b_{c}$, and $\operatorname{prf}_{c}$ semantics are in one-to-one correspondence with the respective SETAF semantics. By Propositions 3 and 8 this result carries over to $c l-p r f, c l-s t b_{a d m}$, and $c l-s t b_{c f}$. We analyze now the translation wrt. the remaining semantics, i.e. naive $e_{c}, c l$-naive, sem $_{c}, s_{g}, c l$-sem and $c l$-stg.

Definition 14. A SETAF is a pair $S F=(A, R)$ where $A$ is finite, and $R \subseteq\left(2^{A} \backslash\{\emptyset\}\right) \times A$ is the attack relation.

Given a SETAF $S F=(A, R), S \subseteq A$ attacks $a$ if there is a set $S^{\prime} \subseteq S$ with $\left(S^{\prime}, a\right) \in R$. $S$ is conflicting in $S F$ if $S$ attacks some $a \in S ; S$ is conflict-free in $S F$, if $S$ is not conflicting in $S F$, i.e. $S^{\prime} \cup\{a\} \nsubseteq S$ for each $\left(S^{\prime}, a\right) \in R$. We write $S_{S F}^{+}=\{a \in A \mid S$ attacks $a\} . a \in A$ is defended by $S$ in $S F$ if for each set $B \subseteq A$ with $(B, a) \in R$, there is some $b \in B$ such that $S$ attacks $b$. With these extended notions the semantics of AFs generalize to SETAFs as follows.

Definition 15. Given a SETAF $S F=(A, R)$, we denote the set of all conflict-free sets in $S F$ as $c f_{s}(S F)$. For $S \in$ $c f_{s}(S F)$, it holds that

- $S \in \operatorname{adm}_{s}(S F)$ if each a $\in S$ is defended by $S$ in $S F$;

- $S \in$ naive $_{s}(S F)$, if $\nexists T \in c f_{s}(S F)$ with $T \supset S$;

- $S \in \operatorname{stg}_{s}(F)$, if $\nexists T \in c f_{s}(F)$ with $T \cup T_{S F}^{+} \supset S \cup S_{S F}^{+}$;

- $S \in \operatorname{sem}_{s}(F)$, if $S \in a d m_{s}(F)$ and $\nexists T \in a d m_{s}(F)$ s.t. $T \cup T_{S F}^{+} \supset S \cup S_{S F}^{+}$.

In order to present the translation from well-formed CAFs to SETAFs we first introduce an equivalent representation via attack-formulas. As in well-formed CAFs arguments with the same claim are indistinguishable in terms of their 

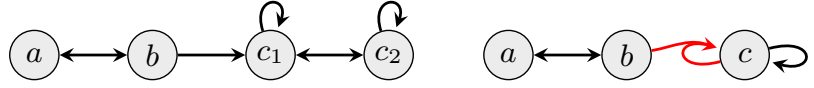

Figure 9: CAF and SETAF from Example 8.

outgoing attacks, we can define attack formulas for each claim $c$. Intuitively, this captures all possible sets of claims which jointly contradict each occurrence of claim $c$.

Definition 16. Given a well-formed $C A F \quad C F=$ $(A, R$, claim $)$, then for each claim $c \in \operatorname{claim}(A)$, the $\mathrm{CNF}-$ attack-formula of $c$ in $C F$ is defined as

$$
\mathcal{C D}_{c}^{C F}=\bigwedge_{a \in A, \operatorname{claim}(a)=c} \bigvee_{(x, a) \in R} \operatorname{claim}(x) .
$$

$\mathcal{D}_{c}^{C F}$ denotes any equivalent DNF-formula over the same set of variables and is called DNF-attack-formula of $c$ in $C F$.

Based on this attack formulas we can define the translation $\mathrm{T}_{c t s}$ mapping well-formed CAFs to SETAFs.

Translation 1. For a well-formed CAF CF= $(A, R$, claim $)$ we define $T_{\text {cts }}(C F)=\left(A^{\prime}, R^{\prime}\right)$ with $A^{\prime}=\operatorname{claim}(A)$ and $R^{\prime}=\left\{(\delta, c) \mid c \in A^{\prime}, \delta \in \mathcal{D}_{c}^{C F}\right\}$.

Theorem 2 ((Dvořák, Rapberger, and Woltran 2020)). $\sigma_{c}(C F)=\sigma_{s}\left(T_{c t s}(C F)\right)$ for each well-formed $C A F C F$ for $\sigma \in\{c f, a d m, p r f, s t b\}$.

We aim to expand these results to the semantics under our consideration. First, we provide examples showing that the correspondence does not hold for for naive ${ }_{c}, \mathrm{sem}_{c}$ and $\mathrm{stg} \mathrm{g}_{c}$.

Example 7. We apply $T_{\text {cts }}$ to $C F$ from Example 2. First observe that $\mathcal{C D}_{x}^{C F}=y \wedge(y \vee z) \wedge z$ and $\mathcal{D}_{x}^{C F}=y \wedge z$, thus $S F=T_{\text {cts }}(C F)=\left(A^{\prime}, R^{\prime}\right)$ with $A^{\prime}=\{x, y, z\}$ and $R^{\prime}=\{(\{y, z\}, x)\}$. Hence, naive $_{s}(S F)=\{\{x, y\},\{x, z\}$, $\{y, z\}\} \neq$ naive $_{c}(C F)=\{\{x\},\{x, y\},\{x, z\},\{y, z\}\}$.

Example 8. We consider the CAF CF from Example 5 with $\operatorname{sem}_{c}(C F)=\operatorname{stg}_{c}(C F)=\{\{b\}\}$. We apply the transformation. The resulting SETAF $S F=T_{c t s}(C F)$ is given in Figure 9. Notice that $\operatorname{sem}_{s}(S F)=\operatorname{stg}_{s}(S F)=\{\{a\},\{b\}\}$.

Next we show that for any claim-set $S$, the translation $\mathrm{T}_{c t s}$ preserves the set $S_{C F}^{+}$of attacked claims.

Lemma 7. Let $C F=(A, R$, claim $)$ be well-formed and $S \subseteq \operatorname{claim}(A)$. Then $S_{C F}^{+}=S_{T_{c t s}(C F)}^{+}$.

Proof. Let $S \subseteq \operatorname{claim}(A)$. By definition, $c \in S_{C F}^{+}$iff $\forall x \in$ $A$ such that $\operatorname{claim}(x)=c$ there is some $b \in S$ such that $(y, x) \in R$ for all $y \in A$ with $\operatorname{claim}(y)=b$. In terms of CNF-attack formulas, $c \in S_{C F}^{+}$iff

$$
\text { for all } \gamma \in \mathcal{C D}_{c}^{C F} \text { it holds that } S \cap \gamma \neq \emptyset \text {. }
$$

Recall that a set $S$ attacks $c$ in $S F=\mathrm{T}_{c t s}(C F)$ if there is some set $S^{\prime} \subseteq S$ such that $\left(S^{\prime}, c\right) \in R$. Rephrasing this property via DNF-attack-formulas yields: $c \in S_{S F}^{+}$iff

$$
\text { exists } \delta \in \mathcal{D}_{c}^{C F} \text { such that } \delta \subseteq S \text {. }
$$

Since (1) is equivalent to (2), the statement follows.
Let $C F$ be a well-formed CAF. By (Dvořák, Rapberger, and Woltran 2020) we have that $\operatorname{adm}_{c}(C F)=$ $a d m_{s}\left(\mathrm{~T}_{c t s}(C F)\right)$ and $c f_{c}(C F)=c f_{s}\left(\mathrm{~T}_{c t s}(C F)\right)$. Since we shift maximization of sets from argument-level to claimlevel, we get that cl-naive $(C F)=$ naive $_{s}\left(\mathrm{~T}_{c t s}(C F)\right)$. By Lemma 7, we have that also the range of extensions is preserved by the translation and thus we get $\sigma(C F)=$ $\sigma_{s}\left(\mathrm{~T}_{c t s}(C F)\right)$ for $\sigma \in\{c l-s e m, c l-s t g\}$.

Theorem 3. For $\sigma \in\{$ sem, naive, stg $\}, C F$ a well-formed $C A F$ and SETAF $S F=T_{c t s}(C F), c l-\sigma(C F)=\sigma_{s}(S F)$.

Overall, we can see that the translations preserve the claim-based semantics and fail to preserve the inherited semantics when they differ from the claim-based semantics.

\section{Expressiveness}

Finally, in this section we investigate the expressiveness of the previously discussed semantics in terms of their signatures, a concept introduced by Dunne et al. (2015) to capture all possible outcomes which can be obtained by AFs when evaluated under a semantics (formally, for a semantics $\sigma$, its (AF-)signature is defined as $\Sigma_{\sigma}^{A F}=\{\sigma(F)$ $F$ is an $\mathrm{AF}\})$. We consider here the analogous claim-based (CAF-)signatures $\Sigma_{\sigma}^{C A F}=\{\sigma(C F) \mid C F$ is a CAF $\}$ and $\Sigma_{\sigma}^{w f}=\{\sigma(C F) \mid C F$ is a well-formed CAF $\}$ with $\sigma$ being either a inherited semantics $\sigma_{c}$ or a claim-based semantics $c l-\sigma$. Note that for any semantics $\sigma$, we have $\Sigma_{\sigma}^{w f} \subseteq \Sigma_{\sigma}^{C A F}$, since each well-formed CAF is indeed a CAF.

Expressiveness of Well-formed CAFs. From the earlier results (see Table 1) we already know that for well-formed CAFs all the considered semantics, except naive $_{c}$, satisfy Imaximality. We show that I-maximality is also sufficient for being realizable in a well-formed CAF.

Theorem 4. Let $\sigma \in\left\{s t b_{c}, c l-s t b_{c f}, c l-s t b_{a d m}\right\}$ and $\tau \in$ $\operatorname{prf}_{c}, c l$-prf, sem $_{c}, c l$-sem, stg, cl-stg, cl-naive $\}$. The following characterizations then hold:

$$
\Sigma_{\sigma}^{w f}=\left\{\mathbb{S} \subseteq 2^{C} \mid \mathbb{S} \text { is I-maximal }\right\} ; \quad \Sigma_{\tau}^{w f}=\Sigma_{\sigma}^{w f} \backslash\{\emptyset\} .
$$

Proof. Recall that $c l$-prf and $p r f_{c}$ coincide on well-formed CAFs (cf. Proposition 3) and so do all three stable variants (cf. Proposition 8). Moreover, in case $s t b_{c}(C F) \neq \emptyset$, $s t b_{c}(C F)=\operatorname{sem}_{c}(C F)=s t g_{c}(C F)$ holds, and by Lemma 6 this extends to $c l-s e m(C F)$ and $c l-s t g(C F)$. By definition of the cl-semantics, I-maximality is thus necessary; the same is true for existence of an extension for all $\tau$-semantics.

By above observation it suffices to provide the realizability step for semantics $p r f_{c}$, stb $b_{c}$, and cl-naive. For $\mathbb{S}=\emptyset$, we construct a CAF $C F=(A, R$, claim $)$ such that $s t b_{c}(C F)=\mathbb{S}$ by just using any $\mathrm{AF}(A, R)$ which has no stable extension. It thus remains to address I-maximality. Let $\mathbb{S}=\left\{S_{1}, \ldots, S_{n}\right\}$ be non-empty and incomparable. We construct $C F=(A, R$, claim) as follows (cf. Example 9):

- $A=\left\{a_{i} \mid a \in S_{i}, 1 \leq i \leq n\right\}$;

- $R=\left\{\left(a_{i}, b_{j}\right) \mid 1 \leq i, j \leq n, a \notin S_{j}\right\}$;

- $\operatorname{claim}\left(a_{i}\right)=a$ for all $1 \leq i \leq n$. 


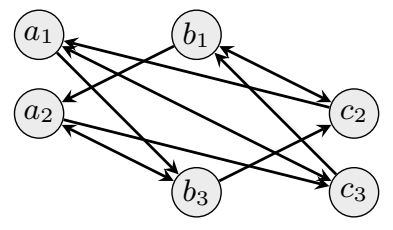

Figure 10: The AF from Example 9.

Note that $C F$ is well-formed. It can be shown that $\operatorname{stb}((A, R))=\operatorname{prf}((A, R))=\left\{\left\{a_{i} \mid a \in S_{i}\right\} \mid S_{i} \in \mathbb{S}\right\}$. $s t b_{c}(C F)=\operatorname{prf}_{c}(C F)=\mathbb{S}$ then follows. Moreover, one can show that also cl-naive $(C F)=\mathbb{S}$.

Example 9. Let $\mathbb{S}=\left\{S_{1}, S_{2}, S_{3}\right\}$ with $S_{1}=\{a, b\}$, $S_{2}=\{a, c\}, S_{3}=\{b, c\}$. The construction in the proof of Theorem 4 yields the $C A F C F=(A, R$, claim) given in Figure 10. It can be verified that $\operatorname{stb}((A, R))=\operatorname{prf}((A, R))=\left\{\left\{a_{1}, b_{1}\right\},\left\{a_{2}, c_{2}\right\},\left\{b_{3}, c_{3}\right\}\right\}$. Hence stb $b_{c}(C F)=\operatorname{prf}_{c}(C F)=\mathbb{S}$. On the other hand, we have naive $((A, R))=\operatorname{stb}((A, R)) \cup\left\{\left\{a_{1}, a_{2}\right\}\right.$, $\left.\left\{b_{1}, b_{2}\right\},\left\{c_{1}, c_{2}\right\}\right\}$, thus naive $_{c}(C F)=\{\{a\},\{b\}$, $\{c\},\{a, b\},\{a, c\},\{b, c\}\}$, while for cl-naive $(C F)$ only the subset-maximal among the $c f_{c}(C F)$ extensions are chosen; i.e. cl-naive $(C F)=\mathbb{S}$.

As we will show next there is no well-formed CAF $C F$ such that naive $(C F)=\mathbb{S}$ with $\mathbb{S}$ as in Example 9, thus making $\Sigma_{\text {naive }}^{w f}$ incomparable to $\Sigma_{c l \text {-naive }}^{w f}$. The following proposition is central for our argument.

Proposition 12. Let $C F=(A, R$, claim $)$ be a well-formed $C A F$. Then, for each $c \in \bigcup_{S \in \text { naive }_{c}(C F)} S$ there is an extension $E \in$ naive $((A, R))$ such that all $a \in A$ with $\operatorname{claim}(a)=c$ are contained in $E$.

Proof. As $c \in \bigcup_{S \in \text { naive }_{c}(C F)} S$, there is an argument with claim $c$ that is not self-attacking in $(A, R)$. As $C F$ is wellformed, the set $\{a \in A \mid \operatorname{claim}(a)=c\}$ is conflict-free in $(A, R)$ and thus contained in some $E \in$ naive $((A, R))$.

Lemma 8. For well-formed CAFs, the set $\mathbb{S}=$ $\{\{a, b\},\{a, c\},\{b, c\}\}$ cannot be realized with inherited naive semantics, i.e. $\mathbb{S} \notin \Sigma_{\text {naive }_{c}}^{\text {. }}$.

Proof. Towards a contradiction assume there is a CAF $C F$ with naive $c(C F)=\mathbb{S}$. By Proposition 12 there are sets $E_{a}, E_{b}, E_{c} \in \operatorname{naive}(C F)$ containing all arguments with claim $a, b$, and $c$ respectively. Let us first assume that all three sets $E_{a}, E_{b}, E_{c}$ are different and have different claim sets, i.e. $\operatorname{claim}\left(E_{a}\right), \operatorname{claim}\left(E_{b}\right), \operatorname{claim}\left(E_{c}\right)$ are mutually distinct. W.l.o.g. we can assume that $\operatorname{claim}\left(E_{a}\right)=\{a, b\}$, $\operatorname{claim}\left(E_{b}\right)=\{b, c\}$ and $\operatorname{claim}\left(E_{c}\right)=\{a, c\}$. That is, (a) there is an argument $b_{i} \in E_{a}$ that is not in conflict with any argument with claim $a$; (b) there is $c_{j} \in E_{b}$ that is not in conflict with any argument with claim $b$; and (c) there is $a_{k} \in E_{c}$ that is not in conflict with any argument with claim c. Now consider the set $\left\{a_{k}, b_{i}\right\}$ which is conflict-free by (a). As $\{a, b, c\} \notin \mathbb{S}$ the set $\left\{a_{k}, b_{i}\right\}$ has a conflict with $c_{j}$. By (c) the conflict has to be between $b_{i}$ and $c_{j}$. However, from (b) we have that $c_{j}$ is not in conflict with $b_{i}$. That is, $\left\{a_{k}, b_{i}, c_{j}\right\} \in c f(C F)$ and thus $\{a, b, c\} \in$ naive $_{c}(C F)$, a contradiction to naive $c(C F)=\mathbb{S}$.

The remaining cases, i.e. (i) $E_{a}, E_{b}, E_{c}$ are different but two of the sets have the same claim-set, and (ii) at least two of the sets $E_{a}, E_{b}, E_{c}$ coincide, can be shown to lead to a contradiction by similar arguments.

Expressiveness of General CAFs. We next show that almost all claim-sets can be realized in arbitrary CAFs with inherited semantics. Interestingly all of these semantics, even naive $_{c}$, are equally powerful for CAFs.

Theorem 5. The following characterizations hold:

$$
\begin{aligned}
\Sigma_{\text {stb }_{c}}^{C A F} & =\left\{\mathbb{S} \subseteq 2^{C} \mid \mathbb{S}=\{\emptyset\} \text { or } \emptyset \notin \mathbb{S}\right\} \\
\Sigma_{\text {naive }_{c}}^{C A F} & =\Sigma_{\text {prf }_{c}}^{C A F}=\Sigma_{\text {sem }_{c}}^{C A F}=\Sigma_{\text {stg }}^{C A F}=\Sigma_{\text {stb }}^{C A F} \backslash\{\emptyset\}
\end{aligned}
$$

Proof. The conditions are necessary, in particular since for any $C F=(A, R$, claim $), \emptyset \in \sigma_{c}(C F)$ implies $\sigma(A, R)=$ $\{\emptyset\}$ and thus $\sigma_{c}((A, R$, claim $))=\{\emptyset\}$.

Now we show that the above conditions are also sufficient by giving an actual construction of a realizing CAF. If $\mathbb{S}=\emptyset$ (this only applies to stable semantics) simply use any AF which has no stable extension. If $\mathbb{S}=\{\emptyset\}$ simply consider the empty $\operatorname{AF}(\emptyset, \emptyset)$. For $\emptyset \notin \mathbb{S}$ construct a $\mathrm{CAF} C F=$ $(A, R$, claim $)$ with $A=\left\{a_{c, S} \mid S \in \mathbb{S}, c \in S\right\}, R=$ $\left\{\left(a_{c, S}, a_{c^{\prime}, S^{\prime}}\right) \mid S, S^{\prime} \in \mathbb{S}, c \in S, c^{\prime} \in S^{\prime}, S \neq S^{\prime}\right\}$ and $\operatorname{claim}\left(a_{c, S}\right)=c$. It holds that naive $c(C F)=s t b_{c}(C F)=$ $\operatorname{prf}_{c}(C F)=\mathbb{S}$. Moreover, since $s t b_{c}(C F) \neq \emptyset$ we have $\operatorname{stb}_{c}(C F)=\operatorname{sem}_{c}(C F)=\operatorname{stg}_{c}(C F)$.

For cl-prf and cl-naive semantics we have that the extension-sets are always I-maximal (see Table 1) and the characterization follows from $\Sigma_{\sigma}^{w f} \subseteq \Sigma_{\sigma}^{C A F}$. For $c l-s t b_{\tau}$, cl-sem and cl-stg we can use the same construction as in the proof of Theorem 5 to show that they are equally expressive as the i-semantics.

Theorem 6. $\Sigma_{c l-p r f}^{C A F}=\Sigma_{c l \text {-naive }}^{C A F}=\Sigma_{c l-p r f}^{w f}, \Sigma_{c l-s t b_{c f}}^{C A F}=$ $\Sigma_{c l \text {-stb }}^{C A b_{a d m}}=\Sigma_{\text {stb }}^{C A F}$ and $\Sigma_{c l \text {-sem }}^{C A F}=\Sigma_{c l \text {-stg }}^{C A F}=\Sigma_{s t b_{c}}^{C A F} \backslash\{\emptyset\}$.

\section{Discussion}

We thoroughly studied semantics for claim-augmented argumentation frameworks. These frameworks are well suited to study aspects of abstract argumentation in connection with instantiation procedures. As we have seen, semantics for such frameworks can be defined in different ways and we have carefully analyzed this effect by showing how these semantics relate to each other and how they relate to SETAFs. We also have obtained a full picture on their expressiveness.

Future work includes a closer look on other semantics; also signatures for conflict-free, admissible, and complete sets remain to be settled. Further, a complexity analysis for the claim-based semantics introduced in this paper is on our agenda, complementing the results in (Dvoráák and Woltran 2020). Moreover, we want to study the properties of CAF semantics by considering structured argumentation, e.g., ABA+ (Bondarenko, Toni, and Kowalski 1993). Finally, it would be worth to investigate the newly introduced semantics in connection with rationality postulates (Caminada and Amgoud 2007; Amgoud and Besnard 2013). 


\section{Acknowledgments}

This research has been supported by WWTF through project ICT19-065, and FWF through projects P30168 and W1255N23.

\section{References}

Amgoud, L., and Besnard, P. 2013. Logical limits of abstract argumentation frameworks. Journal of Applied NonClassical Logics 23(3):229-267.

Atkinson, K.; Baroni, P.; Giacomin, M.; Hunter, A.; Prakken, H.; Reed, C.; Simari, G. R.; Thimm, M.; and Villata, S. 2017. Towards artificial argumentation. AI Magazine 38(3):25-36.

Baroni, P.; Caminada, M.; and Giacomin, M. 2011. An introduction to argumentation semantics. Knowledge Eng. Review 26(4):365-410.

Baroni, P.; Gabbay, D. M.; and Giacomin, M. 2018. Handbook of Formal Argumentation. College Publications.

Baumann, R., and Brewka, G. 2019. Extension removal in abstract argumentation - an axiomatic approach. In The Thirty-Third AAAI Conference on Artificial Intelligence, AAAI 2019, The Thirty-First Innovative Applications of Artificial Intelligence Conference, IAAI 2019, The Ninth AAAI Symposium on Educational Advances in Artificial Intelligence, EAAI 2019, Honolulu, Hawaii, USA, January 27 February 1, 2019, 2670-2677. AAAI Press.

Bondarenko, A.; Toni, F.; and Kowalski, R. A. 1993. An assumption-based framework for non-monotonic reasoning. In Pereira, L. M., and Nerode, A., eds., Logic Programming and Non-monotonic Reasoning, Proceedings of the Second International Workshop, Lisbon, Portugal, June 1993, 171189. MIT Press.

Caminada, M., and Amgoud, L. 2007. On the evaluation of argumentation formalisms. Artif. Intell. 171(5-6):286-310.

Caminada, M.; Sá, S.; Alcântara, J.; and Dvořák, W. 2015a. On the difference between assumption-based argumentation and abstract argumentation. IfCoLog Journal of Logic and its Applications 2(1):15-34.

Caminada, M.; Sá, S.; Alcântara, J.; and Dvořák, W. 2015b. On the equivalence between logic programming semantics and argumentation semantics. Int. J. Approx. Reasoning 58:87-111.

Dung, P. M. 1995. On the acceptability of arguments and its fundamental role in nonmonotonic reasoning, logic programming and n-person games. Artif. Intell. 77(2):321-358. Dunne, P. E.; Dvořák, W.; Linsbichler, T.; and Woltran, S. 2015. Characteristics of multiple viewpoints in abstract argumentation. Artif. Intell. 228:153-178.

Dvořák, W., and Woltran, S. 2020. Complexity of abstract argumentation under a claim-centric view. Artif. Intell. 285.

Dvořák, W.; Rapberger, A.; and Woltran, S. 2020. On the relation between claim-augmented argumentation frameworks and collective attacks. In Proc. ECAI 2020. To appear. available at www.dbai.tuwien.ac.at/research/report/dbai-tr-2020118.pdf.
Gorogiannis, N., and Hunter, A. 2011. Instantiating abstract argumentation with classical logic arguments: Postulates and properties. Artif. Intell. 175(9-10):1479-1497.

Nielsen, S. H., and Parsons, S. 2006. A generalization of Dung's abstract framework for argumentation: Arguing with sets of attacking arguments. In Proc. ArgMAS, volume 4766 of Lecture Notes in Computer Science, 54-73. Springer.

Prakken, H. 2010. An abstract framework for argumentation with structured arguments. Argument and Computation 1(2):93-124.

Rapberger, A. 2020. Defining argumentation semantics under a claim-centric view. In Proc. STAIRS 2020. Accepted for publication - available at https://dbai.tuwien.ac.at/staff/ arapberg/stairs.pdf. 